\title{
Clinical features of cryptococcosis in patients with different immune statuses: a multicenter study in Jiangsu Province-China
}

\author{
Yu Wang ${ }^{1 \dagger}$, Yu Gu ${ }^{2 \dagger}$, Kunlu Shen ${ }^{3 \dagger}$, Xuefan Cui ${ }^{4 \dagger}$, Rui Min ${ }^{4 \dagger}$, Siqing Sun ${ }^{5 \dagger}$, Chunlai Feng ${ }^{6 \dagger}$, Yanbin Chen ${ }^{7 \dagger}$, \\ $\mathrm{Li} \mathrm{Wang}^{8 \dagger}$, Min Cao ${ }^{9 \dagger}$, Jian Yang ${ }^{10 \dagger}$, Jian Yao ${ }^{11}$, Jing Xu ${ }^{12}$, Dang Lin ${ }^{13}$, Yujian Tao ${ }^{14}$, Guoer Ma ${ }^{15}$, Jiaxin Shi ${ }^{16}$,

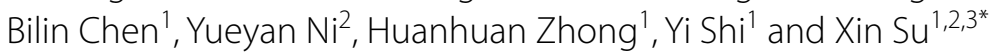

\begin{abstract}
Background: Current guidelines support different management of cryptococcosis between severely immunodeficient and immunocompetent populations. However, few studies have focused on cryptococcosis patients with mild-to-moderate immunodeficiency. We performed this study to determine the clinical features of pulmonary (PC) and extrapulmonary cryptococcosis (EPC) and compared them among populations with different immune statuses to support appropriate clinical management of this public health threat.

Methods: All cases were reported by 14 tertiary teaching hospitals in Jiangsu Province, China from January 2013 to December 2018. The trends in incidence, demographic data, medical history, clinical symptoms, laboratory test indicators, imaging characteristics and diagnostic method of these patients were then stratified by immune status, namely immunocompetent (IC, patients with no recognized underlying disease or those with an underlying disease that does not influence immunity, such as hypertension), mild-to-moderate immunodeficiency (MID, patients with diabetes mellitus, end-stage liver or kidney disease, autoimmune diseases treated with low-dose glucocorticoid therapy, and cancer treated with chemotherapy) and severe immunodeficiency (SID, patients with acquired immunodeficiency syndrome, haematologic malignancies, solid organ transplantation or haematologic stem cell transplantation, idiopathic CD4 lymphocytosis, agranulocytosis, aggressive glucocorticoid or immunosuppressive therapy and other conditions or treatments that result in severe immunosuppression).

Results: The clinical data of 255 cryptococcosis patients were collected. In total, $66.3 \%$ of patients (169) were IC, $16.9 \%$ (43) had MID, and 16.9\% (43) had SID. 10.1\% of the patients (17) with IC were EPC, 18.6\% of the patients (8) with MID were EPC, and $74.4 \%$ of patients (32) were EPC (IC/MID vs. SID, $p<0.001)$. Fever was more common in the SID group than in the IC and MID groups (69.8\% vs. $14.8 \%$ vs. $37.2 \%, \mathrm{p}<0.001)$. Of chest CT scan, most lesions were distributed under the pleura (72.7\%), presenting as nodules/lumps (90.3\%) or consolidations (10.7\%). Pleural effusion was more common in SID group compared to IC group (33.3\% vs. 2.4\%, $\mathrm{p}<0.001)$. Positivity rate on the serum capsular polysaccharide antigen detection ( $\mathrm{CrAg}$ ) test was higher in the SID group than in the other two groups [100.0\% vs.
\end{abstract}

\footnotetext{
*Correspondence: suxinjs@163.com

†Yu Wang, Yu Gu, Kunlu Shen, Xuefan Cui, Rui Min, Siqing Sun, Chunlai

Feng, Yanbin Chen, Li Wang, Min Cao and Jian Yang contributed equally to this paper

${ }^{1}$ Department of Respiratory and Critical Care Medicine, Jinling Hospital,

Medical School of Nanjing University, Nanjing 210002, China

Full list of author information is available at the end of the article
}

(C) The Author(s) 2021. Open Access This article is licensed under a Creative Commons Attribution 4.0 International License, which permits use, sharing, adaptation, distribution and reproduction in any medium or format, as long as you give appropriate credit to the original author(s) and the source, provide a link to the Creative Commons licence, and indicate if changes were made. The images or other third party material in this article are included in the article's Creative Commons licence, unless indicated otherwise in a credit line to the material. If material is not included in the article's Creative Commons licence and your intended use is not permitted by statutory regulation or exceeds the permitted use, you will need to obtain permission directly from the copyright holder. To view a copy of this licence, visit http://creativecommons.org/licenses/by/4.0/. The Creative Commons Public Domain Dedication waiver (http://creativeco mmons.org/publicdomain/zero/1.0/) applies to the data made available in this article, unless otherwise stated in a credit line to the data. 
84.4\% (MID) vs. 78.2\% (IC), $p=0.013]$. Positivity rate on the serum CrAg test was also higher in cryptococcal meningitis patients than in PC patients (100.0\% vs. $79.5 \%, p=0.015)$.

Conclusions: The clinical presentation of MID patients is intermediate between SID and IC patients and is similar to that of IC patients. The serum CrAg test is more sensitive for the identification of SID or EPC patients.

Keywords: Cryptococcosis, Immunocompetent, Immunodeficient, Clinical feature, Mild-to-moderate

\section{Introduction}

The incidence of cryptococcosis in kidney transplant recipients and acquired immunodeficiency syndrome (AIDS) patients is $10.59 / 10,00$ per year. There are one million cases of cryptococcal meningitis globally per year $[1,2]$. Another study found that $32.7 \%$ of patients with cryptococcosis had no recognized underlying diseases [3]. An increasing incidence of pulmonary cryptococcosis $(\mathrm{PC})$ has been identified in immunocompetent patients [4-6].

With regard to Cryptococcus infection, cell-mediated immunity is the predominant defense and is mainly based on Cryptococcus-specific Th1-type CD4+ T cells that produce interleukin-2 (IL-2), tumour necrosis factor (TNF) and interferon (IFN) [7]. A Th1-Th17 cytokine profile is associated with increased phagocytic activity and inhibition of the growth of Cryptococcus [7]. Our previous study revealed that patients with CD4 T lymphocyte cell counts greater than $378 / \mu \mathrm{L}$ were more likely to be asymptomatic and had a lower mortality rate than those with lower CD4+ T lymphocyte counts [8, 9]. Similarly, another study confirmed that patients with CD4+ T lymphocyte counts lower than $400 / \mu \mathrm{L}$ were prone to disseminated cryptococcosis [10]. The mortality rate in patients with low levels of $\mathrm{CD} 4+\mathrm{T}$ lymphocytes was significantly higher than that in patients with normal CD4+ T lymphocyte levels [10, 11]. These reports indicated that immune status significantly influences the clinical features and outcomes of cryptococcosis.

Previous studies have mainly focused on the features of cryptococcal meningitis in severe immunocompromised patients, especially the AIDS population [12, 13]. Few studies have compared the clinical features and the performance of diagnostic methods of cryptococcosis among patients with different immune statuses in one study. The 2010 Infectious Diseases Society of America (IDSA) guidelines recommended managing cryptococcosis patients differently only based on whether they were immunocompetent or severely immunocompromised, such as AIDS and transplant patients. However, there are many patients with mild-to-moderate immunodeficiency for various underlying diseases in the clinic.

The CrAg test is playing an increasingly important role in clinical practice. According to the 2019 Revision and Update of the Consensus Definitions of Invasive
Fungal Disease from the European Organization for Research and Treatment of Cancer and the Mycoses Study Group Education and Research Consortium (EORTC/MSG), a positivity test for CrAg can serve as confirmatory evidence in the diagnosis of cryptococcosis. This greatly improves the diagnostic status of serum CrAg detection.

However, previous studies mostly focused on the population with severe immunodeficiency, and few studies on the patients with immunocompetency and mild-to-moderate immunodeficiency $[4,14]$. The sensitivity of $\mathrm{CrAg}$ in patients with different immune states remains to be further explored.

Are the clinical characteristics of mild-to-moderate immunodeficiency patients more similar to immunocompetent patients or severe immunodeficiency patients? So, based on clinical practice, we conducted this multicentre study and compared the clinical features of patients with different immune statuses.

This study compared the clinical characteristics and the value of the CrAg test in PC and EPC patients who were immunocompetent or immunodeficient (mild-tomoderate or severe immunodeficiency).

\section{Methods}

Proven cryptococcosis cases were reported by 14 tertiary teaching hospitals in Jiangsu Province, China from January 2013 to December 2018. To determine the influence of immune status on the clinical manifestations and diagnostic performance of the $\mathrm{CrAg}$ test, patients were carefully evaluated and divided into three groups: the immunocompetent group (IC group), mildto-moderate immunodeficiency group (MID group) and severe immunodeficiency group (SID group). The patients were also grouped based on whether they had PC or EPC (mainly cryptococcal meningitis). The trend in the incidence and the demographic data, medical history, clinical symptoms, laboratory tests, imaging characteristics and diagnostic methods were analysed and compared (see Fig. 1). The Ethics Committee of Jinling Hospital approved the research protocol. The need of informed consent was waived by the Ethics Committee of Jinling Hospital. None of the data could be traced back to an identifiable patient. 


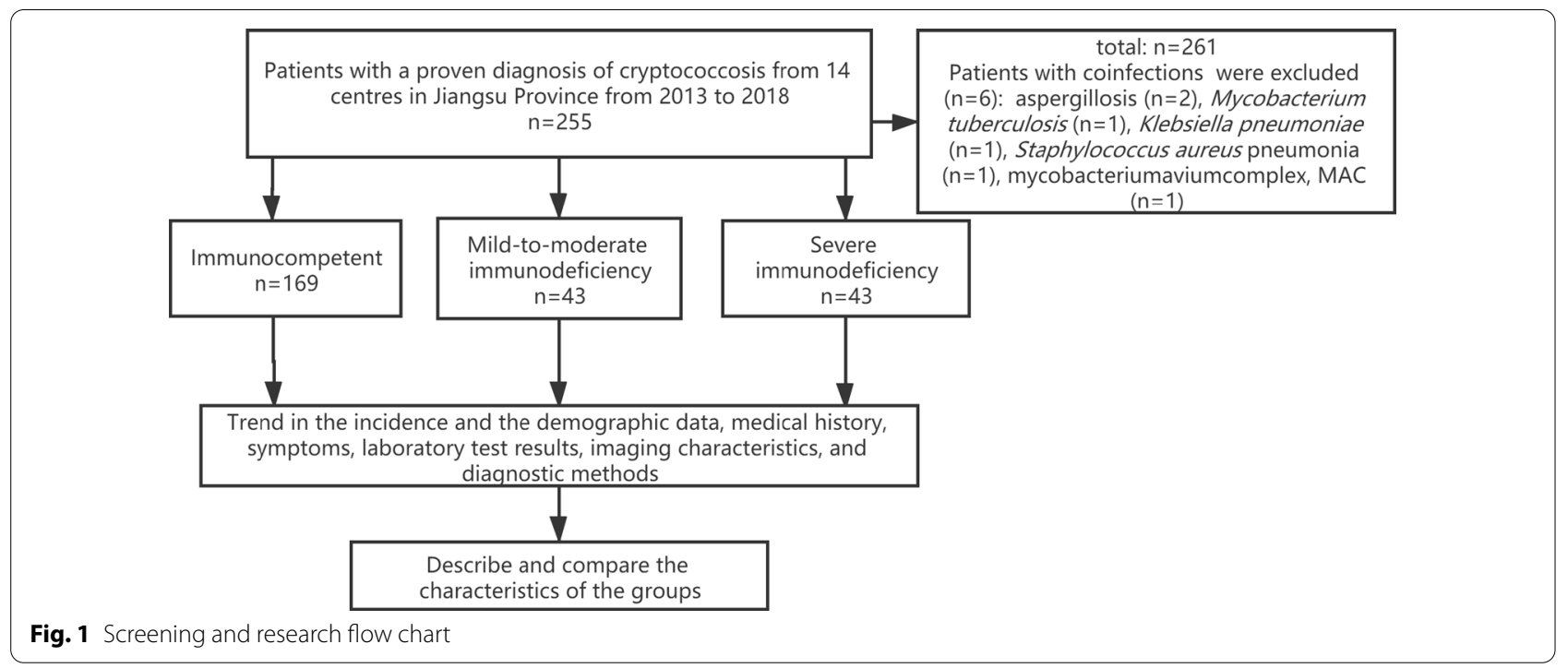

\section{Criteria for diagnosis and grouping Diagnostic and inclusion criteria}

Patients with indicative clinical and imaging manifestations, positive test results for cryptococcal capsular polysaccharide antigen (the name of the kit: $\mathrm{CrAg}$ LFA, CRYPTOCOCCAL ANTIGEN LATERAL FLOW ASSAY; the name of the company/manufacturer: IMMY2701 Corporate Centre Dr Norman Oklahoma 73069 USA-(405) 360-4669; REF: CR2003) in the peripheral blood or cerebrospinal fluid (CSF), or evidence on histopathologic biopsy, or positive results of culture or microscopic examinations [15].

\section{Exclusion criteria}

Patients with co-infections with other pathogens were excluded.

\section{Grouping criteria: \\ 1) Immune status:}

IC patients with no recognized underlying disease or those with an underlying disease that does not influence immunity, such as hypertension.

MID patients with diabetes mellitus, end-stage liver or kidney disease, autoimmune diseases treated with lowdose glucocorticoid therapy, and cancer treated with chemotherapy.

SID patients with AIDS, haematologic malignancies, solid organ transplantation or haematologic stem cell transplantation, idiopathic CD4 lymphocytosis, agranulocytosis, aggressive glucocorticoid or immunosuppressive therapy and other conditions or treatments that result in severe immunosuppression.
The standard of low-dose glucocorticoid is less than $20 \mathrm{mg}$ of prednisone per day, and the cumulative dose is less than $700 \mathrm{mg}$ within 3 weeks before enrollment.

\section{2) Pulmonary and extrapulmonary cryptococcosis:}

$P C$ Lesions were confined to the lung, no dissemination.

$E P C$ Cryptococcal meningitis or cryptococcemia, with or without pulmonary cryptococcosis.

\section{Statistical analysis}

SPSS 26.0 (SPSS Inc., Chicago, IL, USA) were used to perform the statistical analysis and generate the charts in this study. The independent sample T-test was used for continuous variables with normal distributions, and the results are expressed as the means \pm standard deviations. The Mann-Whitney test was used for continuous variables with non-normal distributions, and the results are expressed as the medians and IQRs (interquartile ranges). Univariate analysis of variance and the Brown-Forsythe, Welch and Kruskal-Wallis tests were used for multiple groups. The chi-square test and Fisher's exact test were used for categorical variables. $\mathrm{P}<0.05$ was considered statistically significant, and logistic regression was used as a multivariate analysis to determine the risk factors.

\section{Results}

\section{Incidence trend}

Six patients with co-infections were excluded (aspergillosis, $\mathrm{n}=2$; Mycobacterium tuberculosis, $\mathrm{n}=1$; Klebsiella pneumoniae, $\mathrm{n}=1$; Staphylococcus aureus pneumonia, $\mathrm{n}=1$; mycobacterium complex $[\mathrm{MAC}], \mathrm{n}=1$ ). Finally, 255 patients with confirmed cases were enrolled in the study. The number of newly proven cases are on the 
rise overall $(24,29,48,41,58$ and 55 from 2013 to 2018, respectively) (see Fig. 2).

\section{Demographic data}

In total, $66.3 \%$ of the patients with proven cases (169) were IC, $16.9 \%$ (43) were MID, and $16.9 \%$ (43) were SID. Of the patients with confirmed cases, $62.7 \%$ were males. The mean age was $46.6 \pm 13.7$ years. Patients in the SID group had a lower body mass index (BMI) $[20.2 \pm 2.4 \mathrm{~kg} /$ $\mathrm{m}^{2}$ vs. $23.8 \pm 3.0 \mathrm{~kg} / \mathrm{m}^{2}$ (IC) vs. $24.3 \pm 3.4 \mathrm{~kg} / \mathrm{m}^{2}$ (MID), $\mathrm{p}<0.001$ ], although there was no significant difference between the IC and MID groups. Only $6.3 \%$ of the

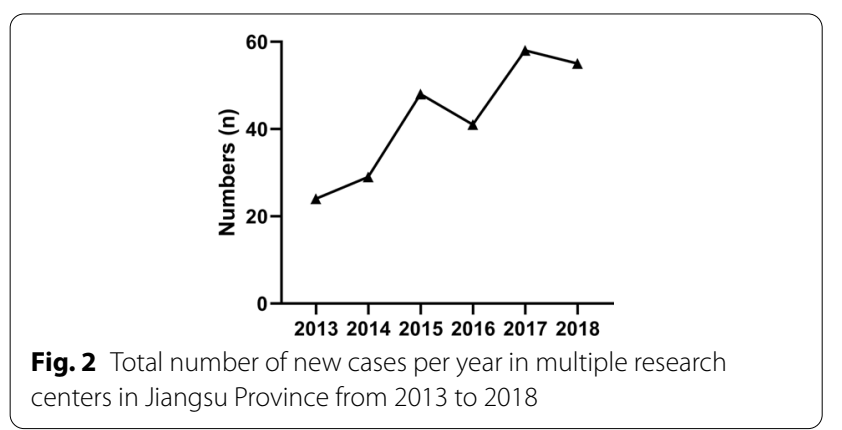

patients had a clear history of exposure to bird or pigeon droppings before onset.

Overall, 77.6\% (198) of the patients had PC, and 22.4\% (57) had EPC. In the IC group, 89.9\% of the patients (152) had PC, and $10.1 \%$ of the patients (17) had EPC. In the MID group, $81.4 \%$ of the patients (35) had PC, and $18.6 \%$ of the patients (8) had EPC. However, in SID group, the majority of patients $(74.4 \%, 32$ cases) were EPC, and $25.6 \%$ of the patients (11) were PC. There was no significant difference in the proportion of patients with MID between the PC and EPC groups (17.2\% vs. 15.9\%, $\mathrm{p}=0.806)$.

\section{Medical history}

The common underlying diseases in MID group were diabetes $(46.5 \%)$, receiving low-dose glucocorticoid therapy (25.6\%), liver dysfunction (18.6\%), chemotherapy (14.0\%), autoimmune diseases (14.0\%), other renal disease $(4.7 \%)$ and haemopathy $(2.3 \%)$ respectively (see Table 1 ).

\section{Clinical symptoms}

The clinical symptoms of cryptococcosis are shown in Fig. 3.

Overall, $27.8 \%(71 / 255)$ of the patients had a fever, and the difference among the IC, MID, and SID groups

Table 1 Demographic data and medical history of cryptococcosis patients with different immune statuses

\begin{tabular}{|c|c|c|c|c|c|}
\hline & Total $\mathrm{N}=255(\%)$ & IC N = $169(\%)$ & MID N=43 (\%) & $\operatorname{SID~N}=43(\%)$ & $P$ value \\
\hline \multicolumn{6}{|l|}{ Demographic data } \\
\hline Sex (male) & $160(62.7)$ & $113(66.9)$ & $24(54.5)$ & $23(54.8)$ & 0.162 \\
\hline Age (y) & $46.6 \pm 13.7$ & $46.4 \pm 12.8$ & $51.8 \pm 14.4$ & $41.9 \pm 14.9$ & $<0.001(\#)$ \\
\hline $\mathrm{BMI}, \mathrm{kg} / \mathrm{m}^{2}$ & $23.3 \pm 3.3$ & $23.8 \pm 3.0$ & $24.3 \pm 3.4$ & $20.2 \pm 2.4$ & $<0.001\left(^{*}\right)$ \\
\hline Contact history & $16(6.3)$ & $12(7.1)$ & $3(7.0)$ & $1(2.3)$ & 0.504 \\
\hline Time since diagnosis(d) & $30.0(14.3,60.0)$ & $30.0(15.0,60.0)$ & $30.0(22.0,60.0)$ & $17.5(9.0,30.0)$ & $<0.001\left(^{*}\right)$ \\
\hline Dissemination & $57(22.4)$ & $17(10.1)$ & $8(18.6)$ & $32(74.4)$ & $<0.001\left(^{*}\right)$ \\
\hline \multicolumn{6}{|l|}{ Medical history } \\
\hline Diabetes 13 (6.4) 8 (14.6) 0.06 & $25(9.8)$ & 0 & $20(46.5)$ & $5(11.6)$ & \\
\hline Glucocorticoid 38 (69.1) & $42(16.5)$ & 0 & $11(25.6)$ & $31(72.1)$ & \\
\hline Liver dysfunction & $11(4.3)$ & 0 & $8(18.6)$ & $3(7.0)$ & \\
\hline Chemotherapy 05 (9.1) & $6(2.4)$ & 0 & $6(14.0)$ & 0 & \\
\hline Autoimmune diseases 027 (49.1) & $18(7.1)$ & 0 & $6(14.0)$ & $12(27.9)$ & \\
\hline Other renal disease 1 (0.5) 4 (7.3) & $8(3.1)$ & 0 & $2(4.7)$ & $6(14.0)$ & \\
\hline Haemopathy & $6(2.4)$ & 0 & $1(2.3)$ & $5(11.2)$ & \\
\hline AIDS 08 (14.6) & $8(3.1)$ & 0 & 0 & $8(18.6)$ & \\
\hline Kidney transplant 013 (23.6) & $13(5.1)$ & 0 & 0 & $13(30.2)$ & \\
\hline Immunosuppressant & $31(12.2)$ & 0 & 0 & $31(72.1)$ & \\
\hline
\end{tabular}

Haemopathy included myelodysplastic syndrome (MDS, $n=2)$, allergic purpura $(n=1)$, multiple myeloma $(M M, n=1)$, lymphoma $(n=1)$, and haemolytic anaemia $(H A, n=1)$; Autoimmune diseases included systemic lupus erythematosus (SLE, $n=10)$, rheumatoid arthritis (RA, $n=3)$, connective tissue disease (CTD, $n=1)$, sarcoidosis $(n=1)$, ankylosing spondylitis $(A S, n=1)$, Hashimoto thyroiditis $(H T, n=1)$, and pemphigoid $(n=1)$; Other renal diseases included nephrotic syndrome (NS, $n=6)$, IgA nephropathy ( $\lg A N, n=1)$, and renal cell carcinoma $(R C C, n=1)$; Liver dysfunction included hepatic cirrhosis ( $n=9)$ and hepatocellular carcinoma (HCC, $\mathrm{n}=2$ )

BMI, body mass index; *SID vs. IC/MID; "MID vs. IC/SID; 


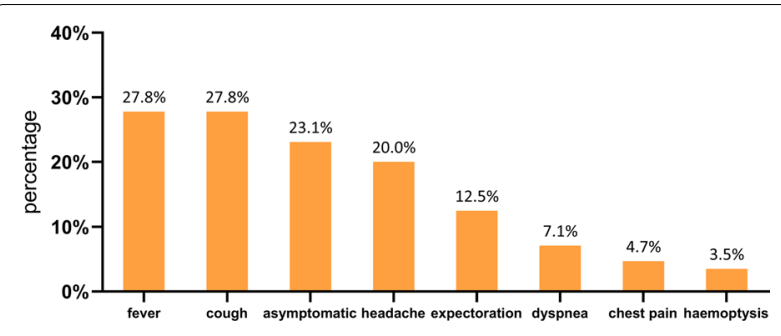

Fig. 3 Distribution of clinical symptoms in 255 patients

was statistically significant ( $14.8 \%$ vs. $37.2 \%$ vs. $69.8 \%$, $\mathrm{p}<0.001)$. Fever was more common in EPC patients than in $\mathrm{PC}$ patients $(75.4 \%$ vs. $14.1 \%, \mathrm{p}<0.001)$.

In total, $23.1 \%(59 / 255)$ of the patients presented as asymptomatic, without significant difference in the proportions among the groups [27.2\% (IC) vs. $14.0 \%$ (MID) vs. $16.3 \%$ (SID), $\mathrm{p}=0.093$ ]. The proportion of patients with asymptomatic infection was higher in the PC group than in the EPC group $(27.3 \%$ vs. $8.8 \%, \mathrm{p}=0.005)$ (see Table 2). The proportion of patients with headache was $88.2 \%$ in the group with cryptococcal meningitis and $3.0 \%$ in PC patients.

\section{Laboratory tests}

Patients in the SID group had lower levels of haemoglobin and albumin than the patients in the other groups $(\mathrm{p}<0.001)$. Inflammatory markers were slightly elevated in the SID group (C-reactive protein, procalcitonin), although there were no significant differences between the IC and MID groups. The mean white blood cell were in the normal range.

The lymphocyte count in the total population was $1.7 \pm 0.9 \times 10^{9} / \mathrm{L}, \quad 2.1 \pm 0.8 \times 10^{9} / \mathrm{L}$ in IC group, $1.5 \pm 0.7 \times 10^{9} / \mathrm{L}$ in MID group, and $0.7 \pm 0.4 \times 10^{9} / \mathrm{L}$ in SID group. The difference between the two groups was statistically significant $(\mathrm{P}<0.001)$ (see Table 3 ).

\section{Imaging characteristics}

The distribution of lesions was classified as solitary $(27.5 \%, 70)$, multiple $(65.5 \%, 167)$ or diffuse $(7.1 \%, 18)$. A subpleural distribution was predominant in all three groups (IC group $72.8 \%$ vs. MID group $64.7 \%$ vs. SID group $83.3 \%, p=0.292$ ), and the remainder were near the hilar or medial belt (see Table 4). The morphology of PC lesions was mainly nodules/lumps (90.3\%) (see Fig. 4).

We present several representative imaging findings in Fig. 5.

Table 2 Clinical symptoms of cryptococcosis patients with different immune statuses

\begin{tabular}{|c|c|c|c|c|c|}
\hline & Total $\mathrm{N}=255(\%)$ & $\mathrm{IC} \mathrm{N}=169(\%)$ & MID N = 43 (\%) & SID N= $43(\%)$ & $P$ value \\
\hline Fever & $71(27.8)$ & $25(14.8)$ & $16(37.2)$ & $30(69.8)$ & $<0.001$ \\
\hline Cough & $71(27.8)$ & $43(25.4)$ & $19(44.2)$ & $9(20.9)$ & $0.027(\#)$ \\
\hline Asymptomatic & $59(23.1)$ & $46(27.2)$ & $6(14.0)$ & $7(16.3)$ & 0.093 \\
\hline Headache & $51(20.0)$ & $17(10.1)$ & $8(18.6)$ & $26(60.5)$ & $<0.001\left(^{*}\right)$ \\
\hline Expectoration & $32(12.5)$ & $22(13.0)$ & $6(14.0)$ & $4(9.3)$ & 0.770 \\
\hline Dyspnea & $18(7.1)$ & $7(4.1)$ & $5(11.6)$ & $6(14.0)$ & $0.036(\&)$ \\
\hline Chest pain & $12(4.7)$ & $8(4.7)$ & $2(4.7)$ & $2(4.7)$ & $>0.999$ \\
\hline Haemoptysis & $9(3.5)$ & $6(3.6)$ & $2(4.7)$ & $1(2.3)$ & 0.833 \\
\hline Meningeal irritation & $6(2.4)$ & 0 & $3(7.0)$ & $3(7.0)$ & $0.008(\&)$ \\
\hline
\end{tabular}

${ }^{*}$ SID vs. IC/MID; ${ }^{\#}$ MID vs. IC/SID; ${ }^{\circledR}$ IC vs. MID/SID

Table 3 Laboratory test indicators of cryptococcosis patients with different immune statuses

\begin{tabular}{|c|c|c|c|c|c|}
\hline & Total N= $255(\%)$ & IC N= $169(\%)$ & MID N = 43 (\%) & $\operatorname{SID~N}=43(\%)$ & $P$ value \\
\hline $\mathrm{Hb}(\mathrm{g} / \mathrm{L})$ & $127.9 \pm 29.4$ & $137.0 \pm 19.5$ & $129.7 \pm 36.9$ & $95.7 \pm 26.6$ & $<0.001$ \\
\hline WBC $\left({ }^{*} 10^{9} / L\right)$ & $7.1 \pm 2.8$ & $7.3 \pm 2.8$ & $6.4 \pm 2.6$ & $7.1 \pm 3.2$ & 0.222 \\
\hline $\operatorname{LY}\left({ }^{*} 10^{9} / \mathrm{L}\right)$ & $1.7 \pm 0.9$ & $2.1 \pm 0.8$ & $1.5 \pm 0.7$ & $0.7 \pm 0.4$ & $<0.001$ \\
\hline $\operatorname{Plt}\left(*^{*} 10^{9} / \mathrm{L}\right)$ & $209.3 \pm 72.7$ & $221.9 \pm 67.3$ & $205.0 \pm 72.5$ & $176.3 \pm 76.5$ & $<0.001\left(^{*}\right)$ \\
\hline CRP (mg/dl) & $2.7(0.5,12.9)$ & $1.3(0.5,8.2)$ & $2.8(0.6,13.8)$ & $14.3(3.8,41.1)$ & $0.044(*)$ \\
\hline PCT $(\mu \mathrm{g} / \mathrm{L})$ & $0.04(0.03,0.06)$ & $0.03(0.02,0.05)$ & $0.03(0.02,0.07)$ & $0.17(0.05,2.40)$ & $0.029\left(^{*}\right)$ \\
\hline Alb (g/L) & $39.6 \pm 7.7$ & $42.7 \pm 4.9$ & $37.3 \pm 7.6$ & $31.5 \pm 9.1$ & $<0.001$ \\
\hline APACHE II & $4.3 \pm 4.7$ & $2.7 \pm 3.2$ & $5.0 \pm 4.5$ & $9.7 \pm 5.5$ & $<0.001$ \\
\hline
\end{tabular}

$\mathrm{Hb}$, haemoglobin; WBC, white blood cells; LY, lymphocyte; Plt, platelets; CRP, C-reactive protein; PCT, procalcitonin; Alb, albumin; APACHE II, acute physiology and chronic health evaluation; CrAg, capsular polysaccharide antigen; *SID vs. IC/MID; ${ }^{*}$ MID vs. IC/SID; ${ }^{\&} I C$ vs. MID/SID; () IQR, interquartile range 
Table 4 Lesion distribution and morphology on imaging of patients with different immune status

\begin{tabular}{|c|c|c|c|c|c|}
\hline & Total $\mathrm{N}=255(\%)$ & IC N=169(\%) & MID N=43(\%) & $\operatorname{SID~N}=43(\%)$ & P value \\
\hline \multicolumn{6}{|l|}{ Distribution (1) } \\
\hline Solitary & $70(27.5)$ & $55(32.5)$ & $10(23.3)$ & $5(11.6)$ & 0.007 (SID vs. IC) \\
\hline Multiple & $167(65.5)$ & $107(63.3)$ & $31(72.1)$ & $29(67.4)$ & 0.534 \\
\hline Diffuse & $18(7.1)$ & $7(4.1)$ & $2(4.7)$ & $9(20.9)$ & $<0.001\left(^{*}\right)$ \\
\hline \multicolumn{6}{|l|}{ Distribution (2) } \\
\hline Subpleural & $157 / 216(72.7)$ & $115 / 158(72.8)$ & $22 / 34(64.7)$ & $20 / 24(83.3)$ & 0.292 \\
\hline Hilar or medial belt & $59 / 216(27.3)$ & $43 / 158(27.2)$ & $12 / 34(35.3)$ & $4 / 24(16.7)$ & \\
\hline \multicolumn{6}{|l|}{ Morphology } \\
\hline Nodule $<1 \mathrm{~cm}$ & $73 / 216(33.8)$ & $53 / 158(31.4)$ & $13 / 34(38.2)$ & $7 / 24(29.2)$ & \\
\hline Nodule/Lump 1-5 cm & $94 / 216(43.5)$ & $61 / 158(38.6)$ & $17 / 34(50.0)$ & $16 / 24(66.7)$ & 0.025 (SID vs. IC) \\
\hline Lump $>5 \mathrm{~cm}$ & $28 / 216(13.0)$ & 26/158 (15.4) & 2/34 (5.9) & 0 & 0.028 (SID vs. IC) \\
\hline Consolidation & $23 / 216(10.7)$ & 20/158 (11.8) & $2 / 34(5.9)$ & $1 / 24(4.2)$ & \\
\hline Cavity & $5 / 216(2.3)$ & $4 / 158(2.4)$ & $1 / 34(2.9)$ & 0 & \\
\hline Tree-in-bud pattern; halo sign & $5 / 216(2.3)$ & $4 / 158(2.4)$ & 0 & $1 / 24(4.2)$ & \\
\hline Pleural effusion & $15 / 216(6.9)$ & 4/158 (2.4) & $3 / 34(8.8)$ & 8/24 (33.3) & $<0.001\left(^{*}\right)$ \\
\hline
\end{tabular}

${ }^{*}$ SID vs. IC/MID

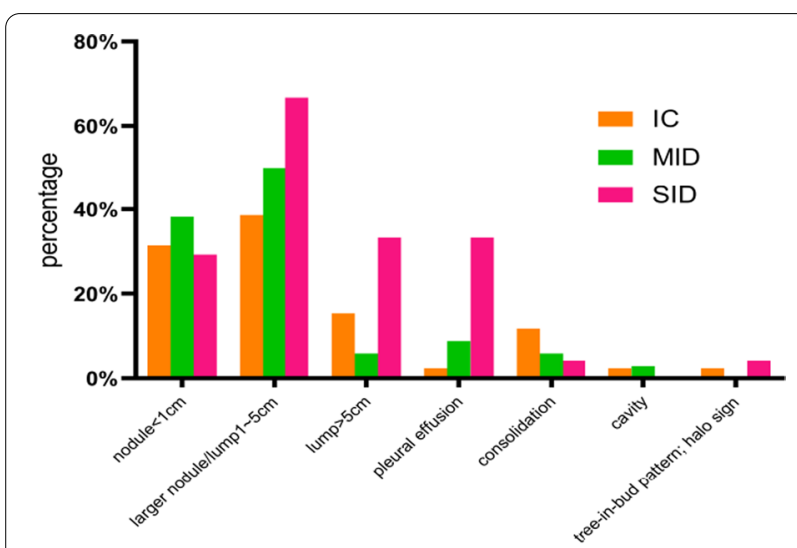

Fig. 4 Leisure morphology on imaging of patients with different immune status

Subpleural nodules were dominant in the IC group, while consolidation shadows and cavities were found in MID group, and more irregular mass shadows and largescale consolidation shadows were found in SID group.

\section{Smear microscopic examination}

The positivity rate of peripheral blood smear microscopic examination in patients with cryptococcosis was $4.5 \%$ (3/66). The positive cases in IC, MID and SID groups accounted for $0 / 21,0 / 15$ and $3 / 30$ (10.0\%) respectively. In patients with cryptococcal meningitis, the positivity rate of cerebrospinal fluid smear microscopic examination was $68.6 \%(35 / 51)$, and positivity rates in IC, MID and SID groups were $35.3 \%(6 / 17), 6 / 8$ and $88.5 \%(23 / 26)$ $[\mathrm{P}<0.001$ (SID vs. IC) $]$ (see Table 5).

\section{CrAg}

The positivity rate of the serum CrAg test in the SID group was higher than those in the other two groups [100.0\% vs. $84.4 \%$ (MID) vs. $78.2 \%$ (IC), $p=0.013$ ]. There was no significant difference between IC and MID group. Among patients with cryptococcal meningitis, the positivity rates of the CSF CrAg test were 100.0\%, 87.5\%, and $100.0 \%$ in the IC, MID and SID groups, respectively $(\mathrm{p}=0.094)$, and the positivity rates of the serum $\mathrm{CrAg}$ test were $100.0 \%$ whatever the immune status. The positivity rate of the serum $\mathrm{CrAg}$ test was also higher in cryptococcal meningitis patients than in PC patients $(100.0 \%$ vs. 79.5\%, $\mathrm{p}=0.015$ ) (see Table 6).

\section{Pathology}

In this study, 174 patients underwent lung histological examination, including 130 cases of percutaneous lung puncture, 31 cases of surgical pneumonectomy, and 13 cases of transbronchial lung biopsy (TBLB), with positivity rates of $90.0 \%(117 / 130), 100 \%(31 / 31)$, and $46.1 \%$ (6/13), respectively (see Table 5).

\section{Discussion}

The number of newly diagnosed cases of cryptococcosis in Jiangsu Province is on the rising. Most of them are patients with immunocompetency. We analyzed the reasons as follows:

Firstly, the sensitivity of serum CrAg test was high compared with galactomannan and 1-3- $\beta$-D-glucan test. The serum sensitivity of patients with normal immune function was $78.2 \%$. A study concluded that the positivity 


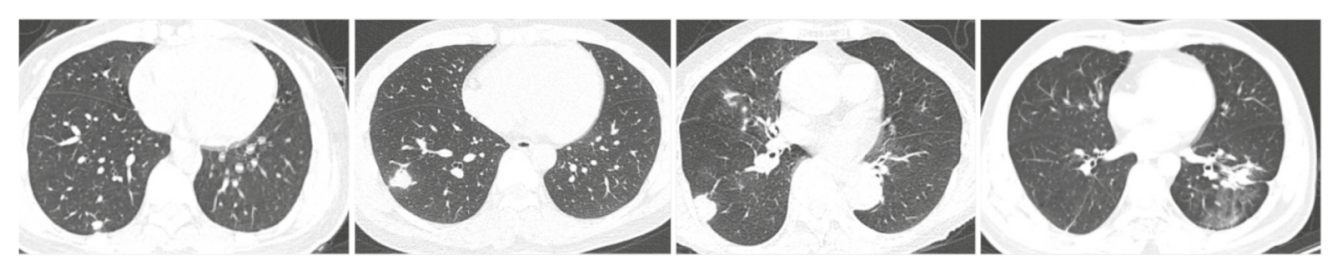

IC: a

b

c

d
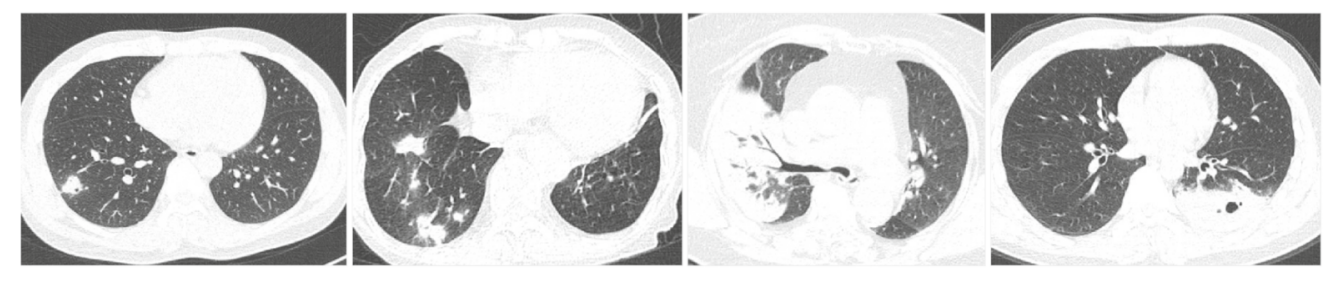

MID: e

f

g

h

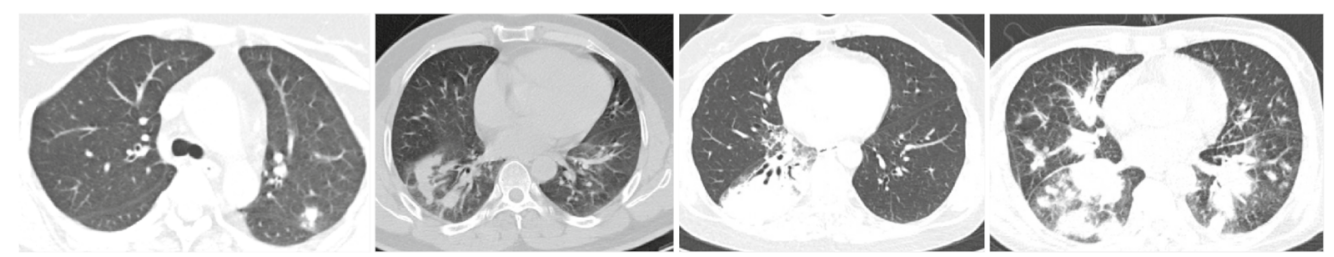

SID: i

j

k

I

Fig. 5 Typical chest computed tomography scans of cryptococcosis patients. IC: a-c: solitary subpleural nodules of varying sizes in the right lower lung $(<3 \mathrm{~cm})$; $\mathbf{d}$ irregular nodular shadows in the left lower lung; MID: e solitary subpleural nodule with a cavity in the right upper lung; $\mathbf{f}$ multiple nodular shadows close to the periphery in the right lung; $\mathbf{g}$ consolidation of the right lung with air bronchogram sign; $\mathbf{h}$ lump in the lower left lung, with a cavity in the middle; SID: i solitary subpleural nodule in the left lung; $\mathbf{j}$ irregular nodular shadows in the right lower lung; $\mathbf{k}$ massive consolidation of the right lung with air bronchogram sign and a small amount of pleural effusion; I lump in the right lower lung with nodules on the other lobes

rate for CrAg in the serum was $80.3 \%$ in IC hosts [16]. The CrAg test is playing an increasingly important role in clinical practice. According to the 2019 EORTC/MSG, a positivity test for CrAg can serve as confirmatory evidence in the diagnosis of cryptococcosis. This greatly improves the diagnostic status of serum CrAg detection. It is widely used as a routine screening for suspected lung infections. With advancements in detection technology and improvements in clinical diagnostic methods, an increasing number of cases of cryptococcosis with immunocompetency have been discovered [17].

Secondly, minimally invasive techniques such as percutaneous pulmonary puncture are gaining popularity. Immunocompetent patients without CNS/bloodstream/ skin involvement were more likely to present as asymptomatic, and lesions were often found on chest CT scan by physical examination [18]. In our study, 27.2\% patients presented with asymptomatic infection. In the past, we usually followed up these patients, and the diagnosis was made when patients with immunocompromised immune system developed extrapulmonary spread or presented with respiratory or systemic symptoms. We can now diagnose early with techniques like percutaneous lung puncture or tracheoscopy. At this stage, the majority of patients are with immunocompetency.

In addition, it is related to the increased understanding and diagnostic awareness of clinicians in recent years. It could also have something to do with the increased incidence of the disease itself.

In this study, $77.6 \%$ of patients had PC, and only $16.9 \%$ of patients had SID. This is inconsistent with the fact that the majority of previously reported cases were CNS Cryptococcus infections. A 10-year (2008-2017) retrospective study from western China concluded that most 
Table 5 Smear, capsular polysaccharide antigen and pathology results of patients with different immune status

\begin{tabular}{|c|c|c|c|c|c|}
\hline & Total N= $255(\%)$ & $\mathrm{IC} \mathrm{N}=169(\%)$ & MID N = $43(\%)$ & SID N=43 (\%) & $P$ value \\
\hline \multicolumn{6}{|l|}{ Smear } \\
\hline Peripheral blood & $3 / 66(4.5)$ & $0 / 21$ & $0 / 15$ & $3 / 30(10.0)$ & 0.544 \\
\hline CSF (meningitis) & $35 / 51(68.6)$ & 6/17 (35.3) & 6/8 (75.0) & 23/26 (88.5) & $<0.001$ (SIDvs.IC) \\
\hline \multicolumn{6}{|l|}{$\mathrm{CrAg}$} \\
\hline Serum & 128/155 (82.6) & 79/101 (78.2) & $27 / 32(84.4)$ & $22 / 22(100.0)$ & $0.048\left(^{*}\right)$ \\
\hline Serum (meningitis) & $21 / 21(100.0)$ & $5 / 5(100.0)$ & $2 / 2(100.0)$ & 14/14 (100.0) & \\
\hline CSF (meningitis) & 44/45 (97.8) & 11/11 (100.0) & $7 / 8(87.5)$ & $26 / 26(100.0)$ & 0.094 \\
\hline Pathology & 147/163 (90.2) & 119/129 (92.3) & $24 / 29(82.8)$ & $4 / 5(80.0)$ & 0.222 \\
\hline $\begin{array}{l}\text { Percutaneous lung } \\
\text { puncture }\end{array}$ & $117 / 130(90.0)$ & 95/104 (91.4) & 18/21 (85.7) & $4 / 5(80.0)$ & 0.551 \\
\hline Pneumonectomy & $31 / 31$ & $25 / 25$ & $5 / 5$ & $1 / 1$ & \\
\hline TBLB & $6 / 13(46.1)$ & $5 / 10(50.0)$ & $1 / 3(33.3)$ & 0 & \\
\hline
\end{tabular}

CSF, cerebrospinal fluid; *SID vs. IC/MID;

Table 6 Clinical characteristics of pulmonary and extrapulmonary cryptococcosis patients

\begin{tabular}{|c|c|c|c|}
\hline & Pulmonary N= $198(\%)$ & Extrapulmonary $\mathrm{N}=57(\%)$ & $P$ value \\
\hline \multicolumn{4}{|l|}{ Site } \\
\hline Only skin & - & $1 / 57(1.8)$ & \\
\hline Only bloodstream & - & $5 / 57(8.8)$ & \\
\hline Only CNS & - & $42 / 57(73.7)$ & \\
\hline Bloodstream and CNS & - & $9 / 57(15.8)$ & \\
\hline \multicolumn{4}{|l|}{ Laboratory test indicators } \\
\hline $\operatorname{LY}\left({ }^{*} 10^{9} / \mathrm{L}\right)$ & $2.0 \pm 0.8$ & $1.0 \pm 0.9$ & $<0.001$ \\
\hline CSF CrAg (meningitis) & - & $44 / 45(97.8)$ & \\
\hline Serum CrAg & 105/132 (79.5) & 23/23 (100.0) & 0.015 \\
\hline \multicolumn{4}{|l|}{ Symptom } \\
\hline Asymptomatic & $54(27.3)$ & $5(8.8)$ & 0.005 \\
\hline Fever & $28(14.1)$ & $43(75.4)$ & $<0.001$ \\
\hline Headache/dizziness & $6(3.0)$ & $45(79.0)$ & $<0.001$ \\
\hline Meningeal irritation & 0 & $6(10.5)$ & $<0.001$ \\
\hline \multicolumn{4}{|l|}{ Medical history } \\
\hline Mild-to-moderate immunodeficiency & $34(17.2)$ & $9(15.9)$ & 0.806 \\
\hline Severe immunodeficiency & $1246(6.1)$ & $3140(54.4)$ & $<0.001$ \\
\hline Glucocorticoid & $17(8.6)$ & $25(43.9)$ & $<0.001$ \\
\hline Immunosuppressants & $10(5.1)$ & $21(36.8)$ & $<0.001$ \\
\hline Autoimmune-related diseases & $6(3.0)$ & $12(21.1)$ & $<0.001$ \\
\hline Kidney transplant & $4(2.0)$ & $9(15.8)$ & $<0.001$ \\
\hline AIDS & $1(0.5)$ & $7(10.5)$ & $<0.001$ \\
\hline Diabetes & $20(10.1)$ & $5(8.7)$ & 0.766 \\
\hline APACHE II, median (IQR) & $2.0(1.0,5.0)$ & $7.0(3.5,10.8)$ & $<0.001$ \\
\hline
\end{tabular}

CNS, central nervous system; LY, lymphocyte

patients with cryptococcosis were immunocompromised $(69.1 \%, 94 / 136)$, and the proportions of patients who were immunocompromised were $55.8 \%(48 / 86)$ in the non-disseminated and $92.0 \%(46 / 50)$ in the disseminated groups, respectively [14]. Another report from Chicago also stated that cryptococcal meningitis was identified in $69.1 \%$ of HIV-infected patients, $34.4 \%$ of non-HIVinfected, nontransplant patients, and $41.9 \%$ of solid organ transplant patients [19]. 
Cryptococcus spores are inhaled primarily through the respiratory tract $[7,20-22]$. They accumulate in the alveoli, causing lung infections and then spreading to the central nervous system, bones, skin, prostate and other organs. This also explains why in our study, the number of patients with $\mathrm{PC}$ was significantly higher than that with EPC.

After the disease, immunodeficient patients cannot produce enough cytokines such as IL-2 and tumor necrosis factor to confine the disease to the lung, and Cryptococcus spores may spread with bloodstream and lymph nodes. The most common site of infection is the central nervous system, which may be caused by the lack of Cryptococcus capsular antibody in CSF, the lack of complement activation system against the capsule, the dopamine in CSF conducive to the growth of Cryptococcus, and may also be related to the increase of the permeability of the tight connection and the destruction of the integrity of the blood-brain barrier [23].

Patients with SID were most likely to be EPC, followed by patients with MID and IC. When extrapulmonary spread occurs, $\mathrm{CrAg}$ sensitivity in serum and CSF can reach almost 100\%. Patients with high load of Cryptococcus pathogens and high antigen concentration in blood are easy to be detected, which is conducive to rapid and definite diagnosis. However, for people with IC and MID, in order to prevent missed diagnosis, negative results cannot completely exclude the diagnosis, and the patient's immune status should be combined.

We conducted a multi-factor analysis to found that patients with severe immune deficiency, headache symptom are independent risk factors for the development of disseminated. But the Odd Ratio (OR) values and confidence intervals $(\mathrm{CI})$ are large. We analyzed the possible reasons as follows: 1 . the proportion of patients with PC and EPC was too large, and the sample size of patients with EPC was small; 2. the observational indicators, especially headache symptoms, were significantly correlated with the outcome and the effect size was large, so the $\mathrm{OR}$ value and $\mathrm{CI}$ were large. When patients with $\mathrm{PC}$ are combined with severe immunodeficiency, headache, central nervous system infection should be considered, and timely lumbar puncture should be performed.

Cryptococcosis mainly occurs in middle-aged men aged 40-50 years, and the male predominance may be related to differences in the immune system and physiology between men and women $[6,24]$.

Based on the above results, more severely immunodeficient patients are more likely to develop a fever. Similarly, EPC patients were significantly more likely to have a fever than PC patients $(75.4 \%$ vs $14.1 \%, \mathrm{p}<0.001)$, and this result was consistent with a study from Western China $(50.0 \%$ vs $23.2 \%, \mathrm{p}=0.001)[14,25,26]$. We believe that when the immunodeficiency is aggravated, the body does not produce a strong immune response and enough cytokine, like interleukin-2 against Cryptococcus infection. Then the pathogen load is high in the body and produce more endogenous pyrogen, through blood circulation effects on the temperature regulating center and change its function, cause fever reaction.

We observed that inflammatory markers, such as CRP protein and PCT, were normal in IC and MID patients and slightly elevated in the SID group. White blood cell were essentially normal in all groups. This suggests that normal inflammatory indicators do not exclude pulmonary cryptococcosis. On the other hand, in the diagnosis and differential diagnosis of disease, if the patient's inflammatory indicators are significantly increased, the presence of other pathogen infection should be further considered.

Multiple subpleural nodules/lumps or consolidations are important imaging features of PC. Patients with SID were more likely to have pleural effusion in this study. These imaging findings have a degree of specificity compared to other pulmonary infections. This also explains why percutaneous lung biopsy is used in most pathological examinations, and the positive rate of percutaneous lung biopsy (90.0\%) is much higher than that of TBLB (46.1\%). These are closely related to the characteristics of cryptocosis in which the lesions are mostly located in subpleural $[12,16$, 27, 28].

The positivity rate of traditional smear microscopic examination was lower than that for the serum $\mathrm{CrAg}$ test. However, in cryptococcal meningitis patients with SID, the positivity rate reached $88.5 \%$ in CSF, which is nearly similar to the $85 \%$ reported in meningitis patients [29]. Therefore, patients with suspected cryptococcal meningitis should be examined by lumbar puncture as soon as possible to obtain a CSF specimen for testing [30, 31].

This study, which uses underlying disease to divide patients into different immune groups, does not seem objective. Part of cases in our study were retrospective, lacking some objective indicators, and there is no single objective indicator to distinguish the systemic immune status of patients. Furthermore, we analyzed the peripheral blood lymphocyte counts of patients and found that the difference in peripheral blood lymphocyte counts between different groups was statistically significant. This also confirms that the classification method we use for underlying diseases is relatively reliable and close to the clinic to facilitate rapid judgment by clinicians. In future studies, if objective immune indicators were used to group patients, such as $\mathrm{CD} 4+\mathrm{T}$ lymphocyte cell count, it might bring us more valuable information. 


\section{Conclusion}

The clinical presentation of MID patients is intermediate between those of SID and IC patients and is similar to those of IC patients in many respects. The serum $\mathrm{CrAg}$ test is relatively more sensitive in SID or EPC patients.

\begin{abstract}
Abbreviations
PC: Pulmonary cryptococcosis; EPC: Extrapulmonary cryptococcosis; IC: Immunocompetent; MID: Mild-to-moderate immunodeficiency; SID: Severe immunodeficiency; CrAg: Capsular polysaccharide antigen; AIDS: Acquired immunodeficiency syndrome; IL-2: Interleukin-2; TNF: Tumor necrosis factor; BMI: Body mass index; IDSA: Infectious Diseases Society of America; EORTC/ MSG: European Organization for Research and Treatment of Cancer and the Mycoses Study Group Education and Research Consortium; Hb: Haemoglobin; WBC: White blood cells; NE: Neutrophil; Plt: Platelets; CRP: C-reactive protein; PCT: Procalcitonin; Alb: Albumin; APACHE II: Acute physiology and chronic health evaluation; BALF: Bronchoalveolar lavage fluid; CSF: Cerebrospinal fluid; TBLB: Transbronchial lung biopsy; CNS: Central nervous system.
\end{abstract}

\section{Acknowledgements}

Thanks to AJE for providing language assistance for this article.

\section{Authors' contributions}

YW, YG, KS, XC, RM, SS, CF and YC analyzed the patient data and contributed in writing this article. LW, MC, JYN, JYO, JX, DL, YT, GM, JS, BC, YN and HZ collected the clinical data. YS and XS contributed in modifying the manuscript. $\mathrm{Xin} \mathrm{Su}$ is the corresponding author. All authors read and approved the final manuscript.

\section{Funding}

This work was supported by the Project of Natural Science Foundation of China (82070011, 81873400), the Key Project of Jiangsu Commission of Health (K2019004), and the "333 project" of Jiangsu Province (BRA2019339).

\section{Availability of data and materials}

The datasets during and/or analysed during the current study are available from the corresponding author on reasonable request.

\section{Declarations}

\section{Ethics approval and consent to participate}

All methods were carried out in accordance with Declaration of Helsinki. The Ethics Committee of Jinling Hospital approved the research protocol. The need of informed consent was waived by the Ethics Committee of Jinling Hospital. None of the data could be traced back to an identifiable patient.

\section{Consent for publication}

Not applicable.

\section{Competing interests}

The authors declare that they have no conflicts of interest.

\section{Author details}

1 Department of Respiratory and Critical Care Medicine, Jinling Hospital, Medical School of Nanjing University, Nanjing 210002, China. ${ }^{2}$ Department of Respiratory and Critical Care Medicine, Jinling Hospital, Nanjing Medical University, Nanjing 210002, China. ${ }^{3}$ Department of Respiratory and Critical Care Medicine, Jinling Hospital, Southern Medical University, Guangzhou 510000, China. ${ }^{4}$ Department of Respiratory and Critical Care Medicine, Jiangsu Province Hospital, Nanjing Medical University, Nanjing 210002, China. ${ }^{5}$ Department of Respiratory and Critical Care Medicine, The Second Hospital of Nanjing, Nanjing 210002, China. ${ }^{6}$ Department of Respiratory and Critical Care Medicine, The Third Affiliated Hospital of Soochow University, Changzhou 213000, China. ${ }^{7}$ Department of Respiratory and Critical Care Medicine, The First Affiliated Hospital of Soochow University, Suzhou 215000, China. ${ }^{8}$ Department of Respiratory and Critical Care Medicine, Nanjing First Hospital, Nanjing Medical University, Nanjing 210002, China. ${ }^{9}$ Department of Respiratory and Critical Care Medicine, Nanjing Drum Tower Hospital, Medical School of Nanjing University, Nanjing 210002, China. ${ }^{10}$ Department of Respiratory and Critical Care Medicine, The Affiliated Jiangning Hospital of Nanjing Medical University, Nanjing 210002, China. ${ }^{11}$ Department of Respiratory and Critical Care Medicine, The First People's Hospital of Nantong Nantong 226000, China. ${ }^{12}$ Department of Respiratory and Critical Care Medicine, Jiangsu Province Hospital of Chinese Medicine, Nanjing 210002, China. ${ }^{13}$ Department of Respiratory and Critical Care Medicine, Suzhou Municipal Hospital, Suzhou 215000, China. ${ }^{14}$ Department of Respiratory and Critical Care Medicine, Affiliated Hospital of Yangzhou University, Yangzhou 225000, China. ${ }^{15}$ Department of Respiratory and Critical Care Medicine, Affiliated Hospital of Jiangsu University, Zhenjiang 212000, China. ${ }^{16}$ Department of Respiratory and Critical Care Medicine, The First People's Hospital of Lianyungang, Lianyungang 222000, China.

Received: 28 March 2021 Accepted: 29 September 2021

Published online: 08 October 2021

\section{References}

1. Chen YT, Lee WS, Tsai CA, Fan WC, Wu PF, Wang FD. Incidence of and risk factors for cryptococcosis in kidney transplant recipients in Taiwan-A nationwide population-based study. Int J Infect Dis. 2019;88:154-8.

2. Gullo FP, Rossi SA, Sardi Jde C, Teodoro VL, Mendes-Giannini MJ, Fusco-Almeida AM. Cryptococcosis: epidemiology, fungal resistance, and new alternatives for treatment. Eur J Clin Microbiol Infect Dis. 2013;32(11):1377-91.

3. Fang LF, Zhang PP, Wang J, Yang Q, Qu TT. Clinical and microbiological characteristics of cryptococcosis at an university hospital in China from 2013 to 2017. Braz J Infect Dis. 2020;24(1):7-12.

4. Liu K, Ding H, Xu B, You R, Xing Z, Chen J, Lin Q, Qu J. Clinical analysis of non-AIDS patients pathologically diagnosed with pulmonary cryptococcosis. J Thorac Dis. 2016;8(10):2813-21.

5. Hou X, Kou L, Han X, Zhu R, Song L, Liu T. Pulmonary cryptococcosis characteristics in immunocompetent patients-A 20-year clinical retrospective analysis in China. Mycoses. 2019;62(10):937-44.

6. Deng H, Zhang J, Li J, Wang D, Pan L, Xue X. Clinical features and radiological characteristics of pulmonary cryptococcosis. J Int Med Res. 2018:46(7):2687-95.

7. Dos Santos VM. Pulmonary cryptococcosis. Arch Iran Med. 2019;22(10):629-30.

8. He Q, Ding Y, Zhou W, et al. Clinical features of pulmonary cryptococcosis among patients with different levels of peripheral blood CD4 ${ }^{+} \mathrm{T}$ lymphocyte counts. BMC Infect Dis. 2017;17(1):768.

9. Thornton CS, Larios O, Grossman J, Griener TP, Vaughan S. Pulmonary Cryptococcus infections as a manifestation of idiopathic CD4 lymphocytopenia: case report and literature review. BMC Infect Dis. 2019;19(1):862.

10. Ding Y, Li P, He Q, Wei H, Wu T, Xia D, Tan M, Shi Y, Su X. The CD4+ T-lymphocyte count is an important predictor for the prognosis of cryptococcosis. Eur J Clin Microbiol Infect Dis. 2017;36(5):897-904.

11. Mukaremera L, Nielsen K. Adaptive immunity to Cryptococcus neoformans infections. J Fungi. 2017;3:64.

12. Hu Z, Chen J, Wang J, et al. Radiological characteristics of pulmonary cryptococcosis in HIV-infected patients. PLoS ONE. 2017;12(3):e0173858.

13. Fujioka K, Nagai T, Kinoshita Y, et al. Successful treatment with voriconazole combined with amphotericin B-liposome for fluconazole-resistant pulmonary cryptococcosis after renal transplantation. CEN Case Rep. 2019:8(4):261-5.

14. Qu J, Zhang X, Lu Y, Liu X, Lv X. Clinical analysis in immunocompetent and immunocompromised patients with pulmonary cryptococcosis in western China. Sci Rep. 2020;10(1):9387.

15. Donnelly JP, Chen SC, Kauffman CA, et al. Revision and update of the consensus definitions of invasive fungal disease from the European Organization for research and treatment of cancer and the mycoses study group education and research consortium. Clin Infect Dis. 2020;71(6):1367-76.

16. Min J, Huang K, Shi C, et al. Pulmonary Cryptococcosis: comparison of Cryptococcal antigen detection and radiography in Immunocompetent and Immunocompromised patients. BMC Infect Dis. 2020;20(1):91.

17. Zhou Y, Lin PC, Ye JR, et al. The performance of serum cryptococcal capsular polysaccharide antigen test, histopathology and culture of the lung 
tissue for diagnosis of pulmonary cryptococcosis in patients without HIV infection. Infect Drug Resist. 2018;11:2483-90.

18. Xie X, Xu B, Yu C, Chen M, Yao D, Xu X, Cai X, Ding C, Wang L, Huang X. Clinical analysis of pulmonary cryptococcosis in non-HIV patients in south China. Int J Clin Exp Med. 2015;8(3):3114-9.

19. Perfect JR, Dismukes WE, Dromer F, Goldman DL, et al. Clinical practice guidelines for the management of cryptococcal disease: 2010 update by the infectious diseases society of America. Clin Infect Dis. 2010;50(3):291-322.

20. Setianingrum F, Rautemaa-Richardson R, Denning DW. Pulmonary cryptococcosis: a review of pathobiology and clinical aspects. Med Mycol. 2019;57(2):133-50.

21. Velagapudi R, Hsueh YP, Geunes-Boyer S, Wright JR, Heitman J. Spores as infectious propagules of Cryptococcus neoformans. Infect Immun. 2009;77:4345-55.

22. Zavala S, Baddley JW. Cryptococcosis. Semin Respir Crit Care Med. 2020;41(1):69-79.

23. Rajesh V, Yen-Ping H, Scarlett G-B, et al. Spores as infectious propagules of Cryptococcus neoformans. Infect Immun. 2009;77:4345-55.

24. Vilchez RA, Irish W, Lacomis J, Costello P, Fung J, Kusne S. The clinical epidemiology of pulmonary cryptococcosis in non-AIDS patients at a tertiary care medical center. Medicine (Baltimore). 2001;80(5):308-12.

25. Wang D, Wu C, Gao J, et al. Comparative study of primary pulmonary cryptococcosis with multiple nodules or masses by $\mathrm{CT}$ and pathology Exp Ther Med. 2018;16(6):4437-44
26. He Q, Ding Y, Zhou W, Li H, Zhang M, Shi Y, Su X. Clinical features of pulmonary cryptococcosis among patients with different levels of peripheral blood CD4+ T lymphocyte counts. BMC Infect Dis. 2017;17(1):768.

27. Liang L, Cong P, Wang Y, Liang Z. Clinical and radiological features of patients with pulmonary cryptococcosis in a hospital of North China. Cureus. 2020;12(5):e8061.

28. Xie LX, Chen YS, Liu SY, Shi YX. Pulmonary cryptococcosis: comparison of CT fndings in immunocompetent and immunocompromised patients. Acta Radiol. 2015;56:447-53.

29. Takazono T, Saijo T, Ashizawa N, et al. Clinical features and cause analysis of false positive results of Aspergillus galactomannan assay in pulmonary cryptococcosis patients. Eur J Clin Microbiol Infect Dis. 2019;38(4):735-41.

30. Rajasingham R, Wake RM, Beyene T, Katende A, Letang E, Boulware DR. Cryptococcal meningitis diagnostics and screening in the era of point-ofcare laboratory testing. J Clin Microbiol. 2019;57(1):e01238-e1318.

31. George IA, Spec A, Powderly WG, Santos CAQ. Comparative epidemiology and outcomes of human immunodeficiency virus (HIV), non-HIV non-transplant, and solid organ transplant associated cryptococcosis: a population-based study. Clin Infect Dis. 2018;66(4):608-11.

\section{Publisher's Note}

Springer Nature remains neutral with regard to jurisdictional claims in published maps and institutional affiliations.
Ready to submit your research? Choose BMC and benefit from:

- fast, convenient online submission

- thorough peer review by experienced researchers in your field

- rapid publication on acceptance

- support for research data, including large and complex data types

- gold Open Access which fosters wider collaboration and increased citations

- maximum visibility for your research: over $100 \mathrm{M}$ website views per year

At BMC, research is always in progress.

Learn more biomedcentral.com/submissions 